\title{
Reviewer 1 Response
}

\author{
Reliable computation of PID gain space for \\ general second order time delay systems \\ Paper ID: TCON-2016-0700
}

July 26, 2016

Dear Reviewer 1

We would like to thank you for your efforts and time in reviewing this paper and also for your helpful comments. The changes made in response to these comments have undoubtedly led to an improved manuscript. We have tried to address comments in the revised manuscript as thoroughly as possible and hope that you will find the changes sufficient and satisfactory.

\section{Detailed response to Reviewer 1 comments}

1. The paper proposes an algorithm for determining the stability region of a PID controller for a second order system with delay. The procedure is based on HermiteBieler, quasi polynomial an Nysuist criterion. The reported examples confirm the validity of the approach. The authors can report also the following paper that presents a similar procedure: "A numerical approach for computing stability region of FOPID controller" $R$. Caponetto and G. Dongola, Journal of the Franklin Institute 350 (2013) 871-889.

Thank you for your comment. The mentioned article has been added to the list of references in the revised version of the paper. 


\title{
Reviewer 2 Response
}

\author{
Reliable computation of PID gain space for \\ general second order time delay systems \\ Paper ID: TCON-2016-0700
}

July 26, 2016

Dear Reviewer 2

We would like to thank you for your efforts and time in reviewing this paper and also for your helpful comments. The changes made in response to these comments have undoubtedly led to an improved manuscript. We have tried to address comments in the revised manuscript as thoroughly as possible and hope that you will find the changes sufficient and satisfactory.

\section{Detailed response to Reviewer 2 comments}

1. This paper is in a well worked area and it is not totally clear what the novelty is to the level required for learned journal publication. There are many similar papers in the area, e.g., Caponetto and coauthor Franklin Institute journal 2013, pp 871-889. The authors need to establish either superiority over such results, i.e., widen the class that can be considered or else examples that clearly demonstrate the merit of the results and techniques in this paper.

Thank you for your comment. As mentioned in the last version of the manuscript, the contributions of the paper can be listed as follows:

First, we have identified and illustrated (in Section 2) an important source of inaccuracy in some of the existing work in the literature.

The second contribution of the manuscript is the novel algorithm which has been proposed for the general class of second order time-delay systems. This algorithm is free from the error cited above, and moreover, can be used for a wider class of system than previously proposed algorithms. To clarify this, a detailed comparison with similar work (with scope of application for a wide class of systems) is outlined below (All work have also been discussed in the paper).

(a) [1]: D-J. Wang. Further results on the synthesis of PID controllers. IEEE Trans. Autom. Control, 52(6):1127-1132, 2007.

(b) [2]: G Martelli. Stability of PID-controlled second-order time-delay feedback systems. Automatica, 45:2718-2722, 2009.

(c) [3]: D-J. Wang and J-H. Zhang. Satbilizing parameters of PID controllers for secondorder plant systems with time-delay. Proceedings of the International Conference on Modelling, Identification and Control, pages 112-117, 2010. Okayama, Japan.

(d) [4]: R. Farkh, K. Laabidi, and M. Ksouri. Stabilizing sets of PI/PID controllers for unstable second order delay system. International Journal of Automation and Computing, pages 210-222, 2014. 
In relation to [1]: On the basis of the discussion presented in Section 2, there are concerns over the accuracy of the algorithm. Moreover, the proposed method in [1] only works for systems with two real poles. Our proposed method does not have such a constraint.

In relation to [2]: The method presented in [2] also only allows real poles for the plant, and moreover our proposed algorithm is significantly computationally cheaper than the approach proposed in [2].

In relation to [3]: Similar to [1].

In relation to [4]: On the basis of the discussion presented in Section 2, there are concerns over the accuracy of this algorithm. Our proposed method works on systems which have stable and unstable poles. However the algorism proposed in [4] can only applied to systems with Real systems with and unstable poles. Moreover the inaccuracy described earlier in section 2 is also applicable in this instance.

With regards to the work by Caponetto and coauthor, while we have added this reference to the revised manuscript, kindly note that it considers first order time-delay systems (not second order). In addition it is based on tuning of fractional order PID controllers. 